\title{
Discovery of X-rays from Mars with Chandra
}

\begin{abstract}
K. Dennerl ${ }^{\star}$
Max-Planck-Institut für extraterrestrische Physik, Giessenbachstraße, 85748 Garching, Germany

Received 16 July 2002 / Accepted 31 July 2002

Abstract. On 4 July 2001, X-rays from Mars were detected for the first time. The observation was performed with the ACIS-I detector onboard Chandra and yielded data of high spatial and temporal resolution, together with spectral information. Mars is clearly detected as an almost fully illuminated disk, with an indication of limb brightening at the sunward side, accompanied by some fading on the opposite side. The morphology and the X-ray luminosity of $\sim 4 \mathrm{MW}$ are fully consistent with fluorescent scattering of solar X-rays in the upper Mars atmosphere. The X-ray spectrum is dominated by a single narrow emission line, which is most likely caused by $\mathrm{O}-\mathrm{K}_{\alpha}$ fluorescence. No evidence for temporal variability is found. This is in agreement with the solar X-ray flux, which was almost constant during the observation. In addition to the X-ray fluorescence, there is evidence for an additional source of $\mathrm{X}$-ray emission, indicated by a faint $\mathrm{X}$-ray halo which can be traced to about three Mars radii, and by an additional component in the X-ray spectrum of Mars, which has a similar spectral shape as the halo. Within the available limited statistics, the spectrum of this component can be characterized by $0.2 \mathrm{keV}$ thermal bremsstrahlung emission. This is indicative of charge exchange interactions between highly charged heavy ions in the solar wind and exospheric hydrogen and oxygen around Mars. Although the observation was performed at the onset of a global dust storm, no evidence for dust-related $\mathrm{X}$-ray emission was found.
\end{abstract}

Key words. atomic processes - scattering - Sun: solar wind - Sun: X-rays, gamma rays - planets and satellites: individual: Mars - X-rays: individual: Mars

\section{Introduction}

During the last years our knowledge about the X-ray properties of solar system objects was considerably enhanced. While the Sun was long known to be an X-ray source (Friedman et al. 1951), X-rays from the Earth (Grader et al. 1968), the Moon (Gorenstein et al. 1974; Schmitt et al. 1991), and Jupiter (Metzger et al. 1983) were detected a few decades later. In 1996 comets were discovered as a new class of X-ray sources (e.g. Lisse et al. 1996; Dennerl et al. 1997; Mumma et al. 1997). Prompted by this discovery, Cravens (2000a) found evidence for X-ray emission from the heliosphere. A marginal $\mathrm{X}$-ray detection of Saturn was reported by Ness \& Schmitt (2000), and, more recently, X-ray emission was discovered from the Galilean satellites Io and Europa and the Io plasma torus (Elsner et al. 2002), and from Venus (Dennerl et al. 2002a). Here the first detection of X-ray emission from Mars is reported.

\section{Observation and data analysis}

Mars was observed on 4 July 2001, during the first opposition after the launch of Chandra. Details about the observing geometry can be found in Table 1 . There were already expectations that Mars would be an X-ray source, though a very faint one (Sect. 4). Thus the prime goal for this observation was to get an unambiguous detection. Direct imaging onto the CCDs of the

\footnotetext{
* e-mail: kod@mpe.mpg.de
}

ACIS-I array, operated in very faint mode, was chosen as the observing mode, in order to get spectral information, which can also be used for suppressing the background efficiently. Due to the high surface brightness of Mars (4.1 mag $\operatorname{arcsec}^{-2}$ ), direct imaging with the more sensitive back-illuminated ACISS3 CCD might have led to problems with contamination by optical light.

The observing technique is illustrated in Fig. 1. Chandra was pointed such that Mars would be close to the nominal aimpoint in I3 during the middle of the observation, to get the sharpest possible image and a minimum of vignetting. As the CCDs were read out every $3.2 \mathrm{~s}$, there was no need for continuous tracking. The photons were individually transformed into the rest frame of Mars, using the geocentric ephemeris of Mars, computed with the JPL ephemeris calculator ${ }^{1}$. Correction for the parallax of Chandra was done with the orbit ephemeris of the delivered data set. For the whole analysis events with Chandra standard grades were used. The ACIS particle background was reduced by screening out events with significant flux in border pixels of the $5 \times 5$ event islands.

In order to avoid contamination of the $\mathrm{X}$-ray signal by unrelated point sources, photons within the point spread function of such sources were removed. Due to the high spatial resolution of the Chandra X-ray telescope, this can be done very efficiently. The insensitive areas, which are created by this method in the celestial reference frame, are very small (cf. Fig. 1). After

\footnotetext{
${ }^{1}$ Available at http://ssd.jpl.nasa.gov/cgi-bin/eph
} 
Table 1. Journal of observations and observing geometry.

\begin{tabular}{cccccccccc}
\hline \hline obsid & date & time & exp time & instrument & $r$ & $\Delta$ & phase & elong & diam \\
& 2001 & {$[\mathrm{UT}]$} & {$[\mathrm{s}]$} & & {$[\mathrm{AU}]$} & {$[\mathrm{AU}]$} & {$\left[{ }^{\circ}\right]$} & {$\left[{ }^{\circ}\right]$} & {$\left[{ }^{\prime}\right]$} \\
\hline 1861 & July 4 & $11: 47: 39-21: 00: 30$ & 33171 & ACIS-I & 1.446 & 0.462 & 18.2 & 153.7 & 20.3 \\
\hline
\end{tabular}

obsid: Chandra observation identifier, exp time: exposure time, $r$ : distance from Sun, $\Delta$ : distance from Earth, phase: angle Sun-Mars-Earth, elong: angle Sun-Earth-Mars, diam: apparent diameter.

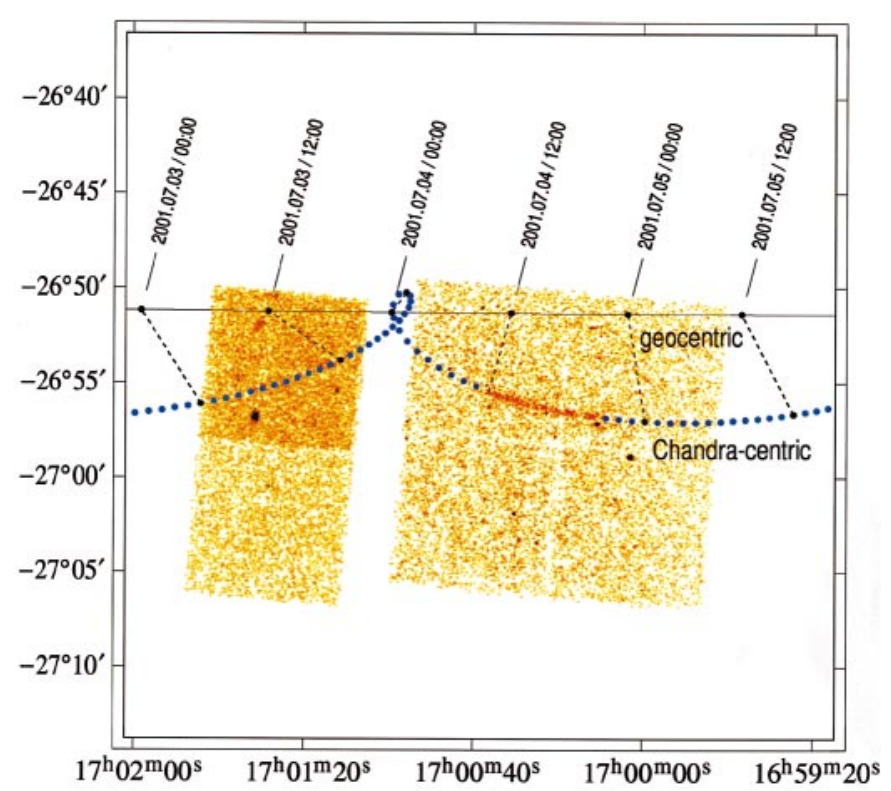

Fig. 1. Apparent motion of Mars in celestial coordinates (J 2000), as seen from Earth and Chandra. The solid line shows the geocentric path of Mars, with its position indicated every 12 hours. Dashed lines illustrate the parallactic shift at these times due to the Chandra orbit. Chandra-centric positions are shown every hour by dots, which match the apparent size of Mars. The loop at 4 July, 0 UT reflects the perigee passage of Chandra. Superimposed on this diagram is the Chandra image in celestial coordinates, obtained at $E<1.0 \mathrm{keV}$ from all events with standard grades. The sequence of dots in the Chandra-centric curve is interrupted to show the curved soft X-ray trail of Mars on the ACIS-I array. In addition to Mars, several point sources show up in the Chandra image. The photons from these sources were excluded in the subsequent analysis.

transformation into the rest frame of Mars, they become diluted along the proper motion direction of Mars, causing an almost negligible reduction of the effective exposure along such streaks. In order to avoid inhomogeneous sensitivity caused by the gaps between the CCDs along the path of Mars (Fig. 1), the analysis was restricted to photons within $100^{\prime \prime}$ from the center of Mars.

\section{Observational results}

\subsection{Morphology}

Mars is clearly detected in the Chandra image (Fig. 2), and, although the photon statistics are low, some general information about the brightness distribution across the disk can be derived. Figure $3 \mathrm{~b}$ shows the average surface brightness as a function

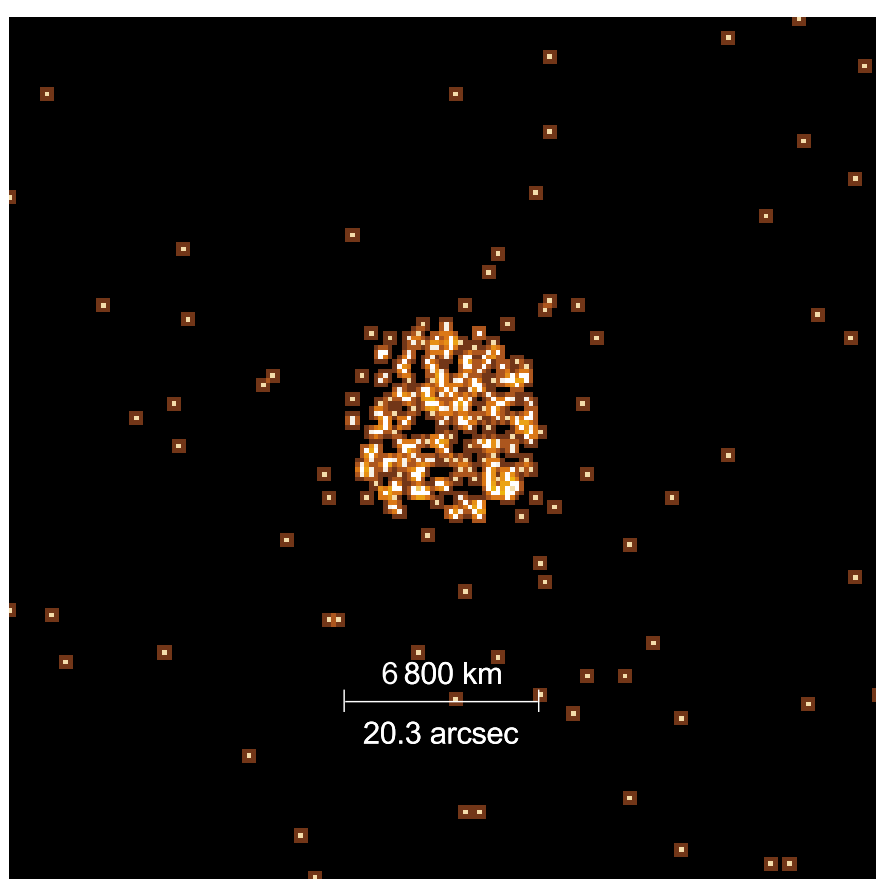

Fig. 2. First X-ray image of Mars, obtained with Chandra ACIS-I on 4 July 2001. Only photons in the instrumental energy range $E=0.40-$ $0.73 \mathrm{keV}$ were selected and transformed into the rest frame of Mars. Trails of point sources were removed.

of the distance from the center of Mars. In the (instrumental) energy range $0.2-1.5 \mathrm{keV}$, a limb brightening by $\sim 25 \%$ is indicated on the sunward limb (Fig. 3b, "dayside"), while a darkening is seen at the opposite limb ("nightside").

At larger distances, the radial brightness profiles show some evidence for a soft X-ray halo around Mars, extending out to $\sim 30$ " ( 3 Mars radii), both on the "dayside" and the "nightside". No evidence for such a halo is seen above $1.5 \mathrm{keV}$. The halo is most pronounced in the energy range $0.5-1.0 \mathrm{keV}$, where the annulus between $r=11^{\prime \prime}$ and $r=30^{\prime \prime}$ around Mars contains $34.6 \pm 8.4$ excess counts relative to the background expected for this area, as determined from the $r=50^{\prime \prime}$ to $r=100^{\prime \prime}$ annulus around Mars.

\subsection{Spectrum}

The X-ray spectra of Mars and its halo are shown in Fig. 4. For the spectrum of Mars photons were extracted within $r<11^{\prime \prime}$ around its center, for the X-ray halo photons within $11^{\prime \prime}<r<$ $30^{\prime \prime}$ were used, and the background was taken in both cases from an annulus around Mars with 50" $<r<100^{\prime \prime}$ (cf. Fig. 3). 

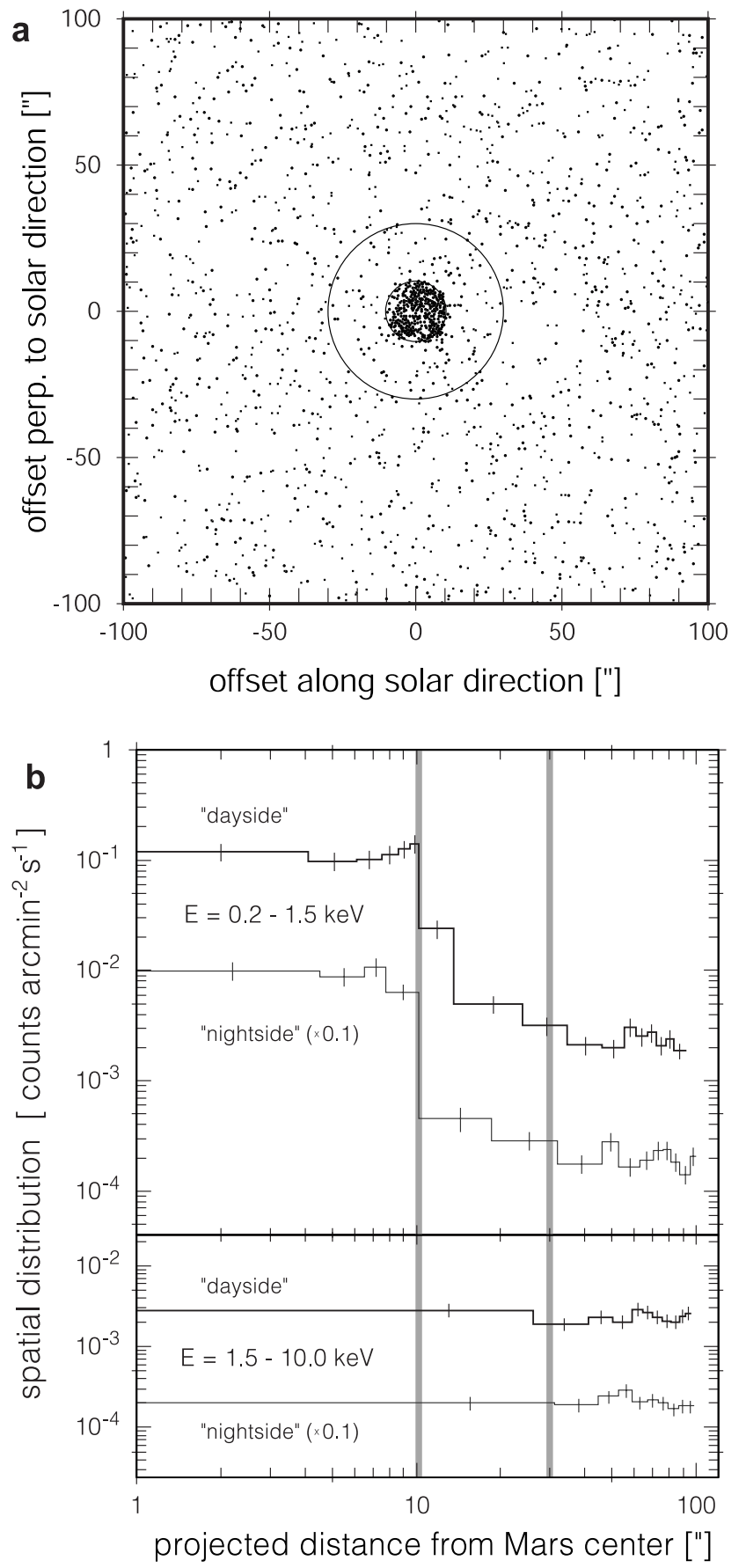

Fig. 3. Spatial distribution of photons around Mars in the ACIS-I observation, after removing the trails of point sources. a) All photons in the energy range $0.2-10.0 \mathrm{keV}$; those with $E \leq 1.5 \mathrm{keV}$ are marked with large dots, while photons with $E>1.5 \mathrm{keV}$ are plotted as small dots. In some cases the dots have been slightly shifted (by typically less than $1^{\prime \prime}$ ) to minimize overlaps. The inner circle, with $r=10$ ' 2 , marks the geometric size of Mars, while the outer one, at $r=30^{\prime \prime}$, illustrates the extent of the soft X-ray halo. b) Spatial distribution of the photons in the soft $(E=0.2-1.5 \mathrm{keV})$ and hard $(E=1.5-10.0 \mathrm{keV})$ energy range, in terms of surface brightness along radial rings around Mars, separately for the "dayside" (offset along projected solar direction $>0$ ) and the "nightside" (offset $<0$ ); note, however, that the phase angle was only $18^{\circ} .2$. For better clarity the nightside histograms were shifted by one decade downward. The bin size was adaptively determined so that each bin contains at least 28 counts. The thick vertical lines mark the radii $10{ }^{\prime} 2$ and $30^{\prime \prime}$ of the circles in a).

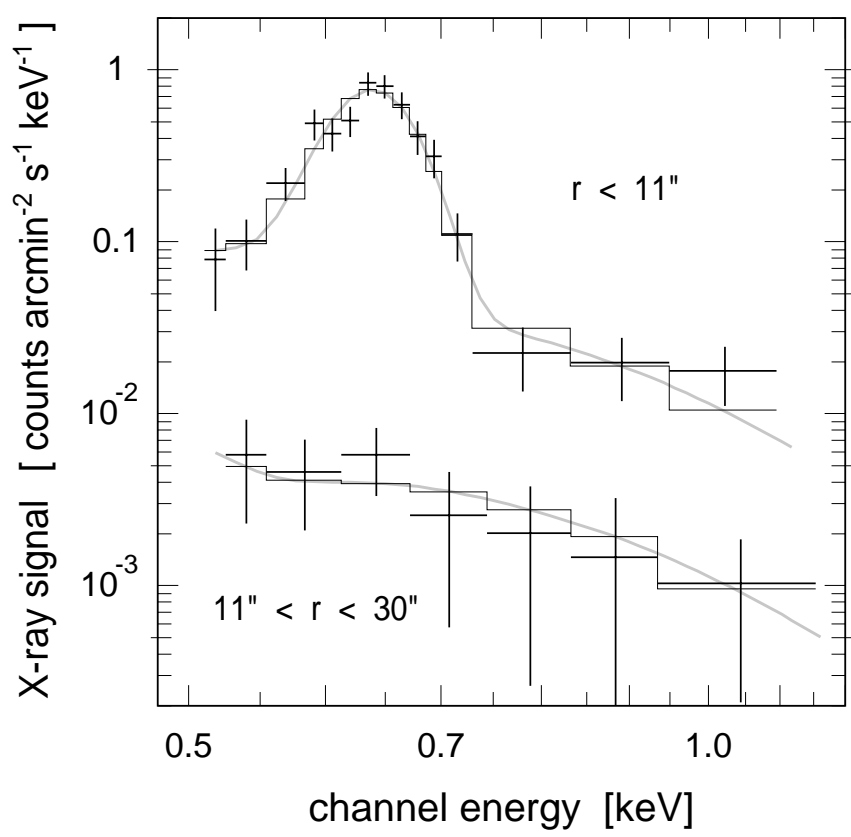

Fig. 4. X-ray spectra of Mars (top) and its X-ray halo (bottom). Crosses with $1 \sigma$ error bars show the observed spectra; the model spectra, convolved with the detector response, are indicated by grey curves (unbinned) and by histograms (binned as the observed spectra). The spectrum of Mars itself is characterized by a single narrow emission line (this is most likely the $\mathrm{O}-\mathrm{K}_{\alpha}$ fluorescence line; see the text for a discussion of the apparent displacement of the line energy). At higher energies the presence of an additional spectral component is indicated. The spectral shape of this component can be well modeled by the same $0.2 \mathrm{keV}$ thermal bremsstrahlung emission which describes the spectrum of the X-ray halo.

The ACIS-I spectrum of Mars at energies below $E \sim$ $0.8 \mathrm{keV}$ can be well described $\left(\chi_{v}^{2}=0.95\right.$ for $10 \mathrm{de}-$ grees of freedom) by a single Gaussian emission line at $E=0.65 \pm 0.01 \mathrm{keV}$ with $\sigma=20 \pm 10 \mathrm{eV}$ (i.e., not significantly broadened) and a flux of $(6.3 \pm 0.8) \times$ $10^{-5} \mathrm{ph} \mathrm{cm}^{-2} \mathrm{~s}^{-1}$. Above energies of $\sim 0.8 \mathrm{keV}$, the presence of an additional component is indicated. In the (instrumental) energy range $E=0.5-1.1 \mathrm{eV}$ (Fig. 4), the spectrum can be well modeled $\left(\chi_{v}^{2}=0.89\right.$ for 12 degrees of freedom) by a single Gaussian emission line at $E=0.65 \pm$ $0.01 \mathrm{keV}$, only instrumentally broadened $(\sigma \equiv 0 \mathrm{eV})$, with a flux of $(5.4 \pm 0.9) \times 10^{-5} \mathrm{ph} \mathrm{cm}^{-2} \mathrm{~s}^{-1}$, superimposed on thermal bremsstrahlung with $k T$ fixed to $0.2 \mathrm{keV}$ (as for the halo; see below), which contributes a flux of $(1.5 \pm 0.4) \times$ $10^{-5} \mathrm{ph} \mathrm{cm}^{-2} \mathrm{~s}^{-1}$, or $(1.5 \pm 0.4) \times 10^{-14} \mathrm{erg} \mathrm{cm}^{-2} \mathrm{~s}^{-1}$ in the energy range $0.5-1.2 \mathrm{keV}$.

The X-ray halo can be well characterized by thermal bremsstrahlung emission with $k T=0.2 \pm 0.1 \mathrm{keV}$ and a flux of $(0.9 \pm 0.4) \times 10^{-5} \mathrm{ph} \mathrm{cm}^{-2} \mathrm{~s}^{-1}$, or $(0.9 \pm 0.4) \times$ $10^{-14} \mathrm{erg} \mathrm{cm}^{-2} \mathrm{~s}^{-1}$ in the energy range $0.5-1.2 \mathrm{keV}$. Further spectral analysis is limited by the poor photon statistics.

\subsection{Temporal variability}

The X-ray flux from Mars was fairly constant during the whole observation, at $(9.3 \pm 0.6) \times 10^{-3}$ counts $^{-1}$ for 


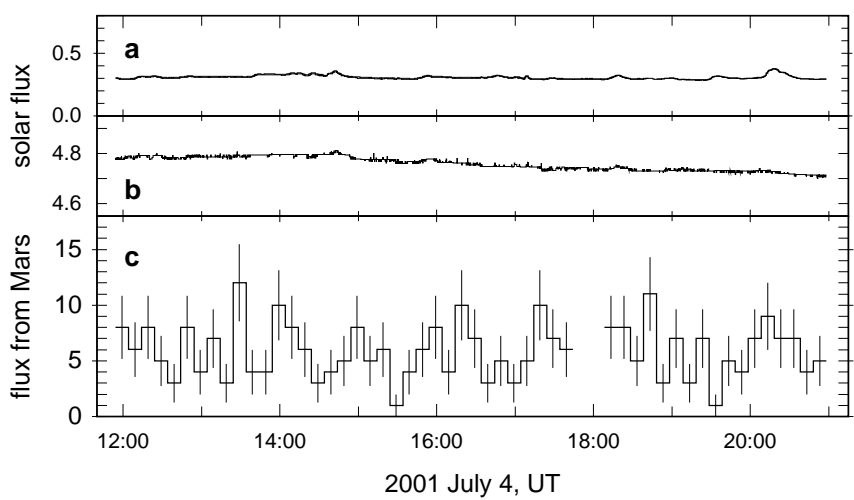

Fig. 5. Temporal behaviour of the soft $X$-ray flux from the Sun and Mars. a) $1-8 \AA$ ( $1.55-12.4 \mathrm{keV})$ solar flux in $10^{-3} \mathrm{erg} \mathrm{cm}^{-2} \mathrm{~s}^{-1}$ at $1.0 \mathrm{AU}$, as measured with GOES-8 and GOES-10. b) $1-500 \AA$ $(0.025-12.4 \mathrm{keV})$ solar flux in $10^{10}$ photons $\mathrm{cm}^{-2} \mathrm{~s}^{-1}$ scaled to $1.0 \mathrm{AU}$, as measured with $\mathrm{SOHO} / \mathrm{SEM}$. The times in a) and b) were shifted by $+458 \mathrm{~s}$, to take the light travel time delay between Sun $\rightarrow$ Mars $\rightarrow$ Chandra and Sun $\rightarrow$ SOHO/GOES into account. c) X-ray flux from Mars as observed with Chandra ACIS-I, in counts/bin, shown with a bin size of $600 \mathrm{~s}$, and derived by extracting all photons below $1 \mathrm{keV}$ from a circle of $11^{\prime \prime} 0$ radius centered at Mars. The interruption at 18:00 UT is caused by Mars crossing the gap between CCD I3 and I1. With $\sim 0.1$ background events per time bin the background is negligible (cf. Fig. 3).

$E<1 \mathrm{keV}$ (Fig. 5c). According to the Kolmogorov-Smirnov test, the probability that the observed count rates are statistical fluctuations around a constant value is $30 \%$; the significance for intrinsic variability is only $1.3 \sigma$. The solar X-ray flux, monitored simultaneously with GOES-8 and GOES-10 (Fig. 5a) and SOHO/SEM (Fig. 5b) was also quite constant, and unusually low for this phase in the solar cycle (Fig. 13). These satellites observed within $8^{\circ}$ the same solar hemisphere which was irradiating Mars (Fig. 6).

\section{Modeling the X-ray appearance of Mars}

It was expected that fluorescent scattering of solar X-rays in the atmosphere would be the dominant source of the X-ray radiation from Venus and Mars. In order to get a reliable prediction about the X-ray properties of these planets, a numerical model was developed for computing simulated images in the individual fluorescence lines. This model was already successfully applied to the Chandra observation of Venus (Dennerl et al. 2002a). For Mars, it was used in order to optimize the time of the observation (Sect. 4.5). The ingredients to the model are the composition and density structure of the Mars atmosphere, the photoabsorption cross sections and fluorescence efficiencies of the major atmospheric constituents, and the incident solar spectrum.

\subsection{Mars atmosphere}

For the Mars atmosphere a simplified model was adopted, which describes the total density $\rho$ in the form of analytical expressions for heights $0-100 \mathrm{~km}$ (Sehnal 1990a) and 100-1000 km (Sehnal 1990b). In order to get a smooth

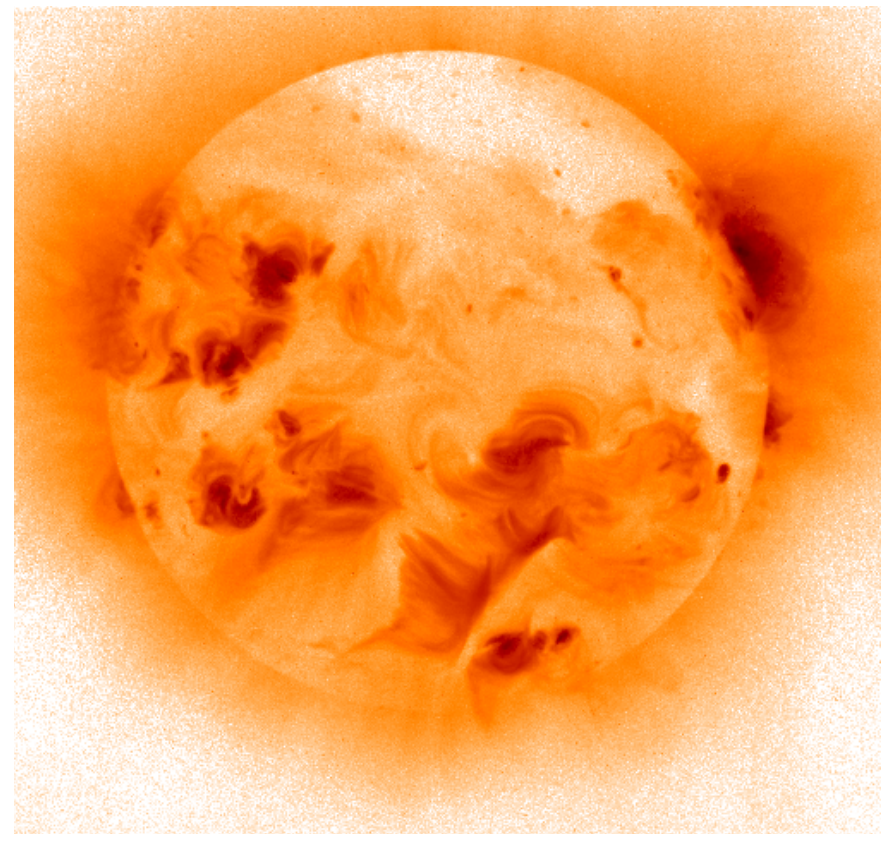

Fig. 6. Soft X-ray image of the Sun on 4 July 2001, 14:22 UT, obtained with Yohkoh SXT in the energy range 0.25-4.0 keV (http://mssly4.mssl.ucl.ac.uk/ydac/). It shows, within $8^{\circ}$, the same solar hemisphere which was irradiating Mars.

transition between both regions, the density at $100-135 \mathrm{~km}$ heights was computed with both methods, and the $\log \rho$ values were weighted according to their distance from 100 and $135 \mathrm{~km}$. The analytical expressions are given for solar minimum, solar maximum, and the intermediate state. For the simulation, the solar maximum conditions were selected, motivated by the general behaviour of the soft solar X-ray flux (Fig. 13). For simplicity it was assumed that the Mars atmosphere is composed of $\mathrm{C}, \mathrm{N}$, and $\mathrm{O}$ only, neglecting the $\sim 1.6 \%$ contribution of other elements, mainly $\mathrm{Ar}^{2}$, and the following composition was adopted: $64.9 \%$ oxygen, $32.4 \%$ carbon and $2.7 \%$ nitrogen. As the main constituents, $\mathrm{C}$ and $\mathrm{O}$, are contained in $\mathrm{CO}_{2}$ (which accounts for more than $95 \%$ of the Mars atmosphere), this composition was assumed to be homogeneous throughout the atmosphere.

\subsection{Photoabsorption cross sections}

The values for the photoabsorption cross sections were taken from Reilman \& Manson (1979), supplemented by the following $K$-edge energies (see Dennerl et al. 2002a for a discussion of these energies): $E_{K_{\mathrm{C}}}=296.1 \mathrm{eV}, E_{K_{\mathrm{N}}}=409.9 \mathrm{eV}$, $E_{K_{\mathrm{O}}}=544.0 \mathrm{eV}$. From these values and the $\mathrm{C}, \mathrm{N}$, and $\mathrm{O}$

\footnotetext{
2 Argon would produce a $\mathrm{K}_{\alpha}$ fluorescence line at $2.96 \mathrm{keV}$ with a fluorescence yield $y_{\mathrm{Ar}}=0.118$ (Krause 1979), which exceeds that of nitrogen by a factor of 20 . The photoabsorption cross section of Ar$\mathrm{K}_{\alpha}$ is $15 \%$ of that of $\mathrm{N}-\mathrm{K}_{\alpha}$, and there are about half as many Ar atoms as $\mathrm{N}$ atoms, so that the $\mathrm{X}$-ray albedo of Mars in the $\mathrm{K}_{\alpha}$ fluorescence lines of $\mathrm{N}$ and $\mathrm{Ar}$ should be comparable. The incident solar flux at $3.0 \mathrm{keV}$, however, is many orders of magnitude lower than at $0.4 \mathrm{keV}$ (Fig. 9b), consistent with the non-detection of any signal from Mars at $3.0 \mathrm{keV}$.
} 


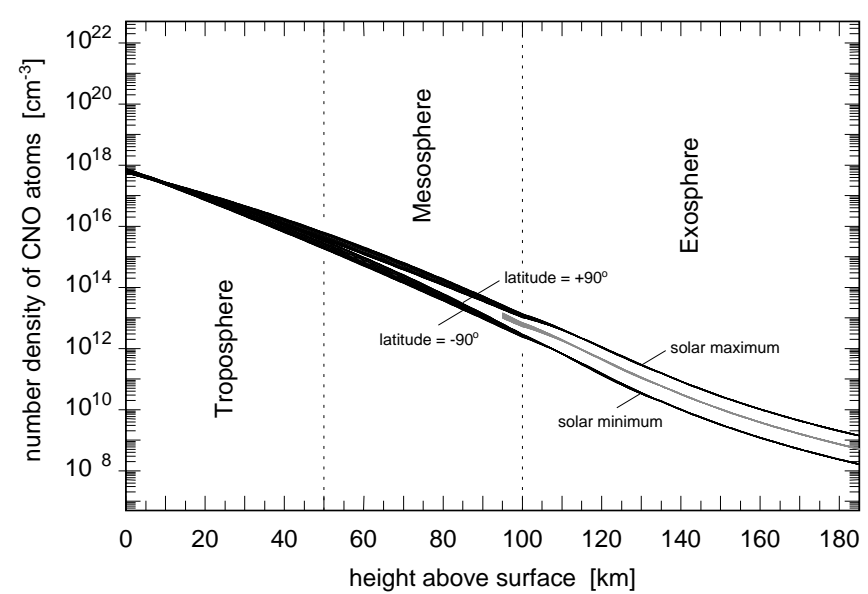

Fig. 7. Number density $n_{\mathrm{CNO}}=n_{\mathrm{C}}+n_{\mathrm{N}}+n_{\mathrm{O}}$ of the sum of $\mathrm{C}, \mathrm{N}$, and $\mathrm{O}$ atoms in the Mars model atmosphere as a function of the height above the surface. Three sets of curves are shown: for solar minimum, solar maximum, and the intermediate state (only above $95 \mathrm{~km}$ height, for better clarity). Below $100 \mathrm{~km}$, the density depends also on latitude.

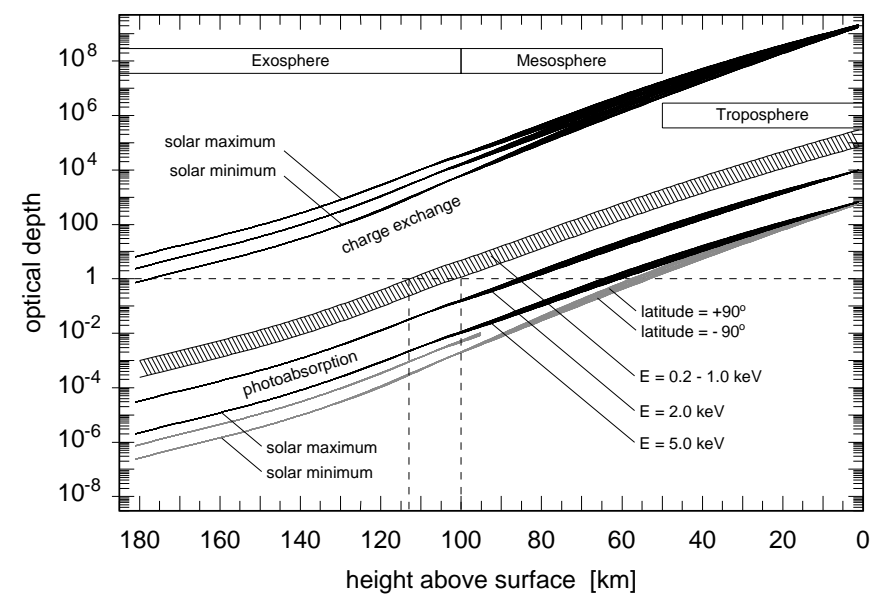

Fig. 8. Optical depth $\tau=\tau_{\mathrm{C}}+\tau_{\mathrm{N}}+\tau_{\mathrm{O}}$ of the Mars model atmosphere with respect to charge exchange (above) and photoabsorption (below), as seen from outside. The upper/lower boundaries of the hatched area refer to energies just above/below the $\mathrm{C}$ and $\mathrm{O}$ edges (cf. Fig. 9a). For better clarity the dependence of the photoabsorption on the solar cycle is only shown for $E=5.0 \mathrm{keV}$; the curves for the other energies refer to solar maximum. The dashed horizontal line, at $\tau=1$, marks the transition between the transparent $(\tau<1)$ and opaque $(\tau>1)$ region. For charge exchange interactions a constant cross section of $3 \times 10^{-15} \mathrm{~cm}^{2}$ was assumed. Due to this high cross section, $\tau=1$ is reached already at heights of $180 \mathrm{~km}$ and above, while for photoabsorption at $E=0.2-1.0 \mathrm{keV}$ the atmosphere becomes opaque between $113 \mathrm{~km}$ and $100 \mathrm{~km}$, for solar maximum conditions. During solar minimum, this transition occurs $\sim 10 \mathrm{~km}$ deeper in the atmosphere.

contributions listed above, the effective photoabsorption cross section of the Mars atmosphere was computed (Fig. 9a). This, together with the atmospheric density structure, yielded the optical depth of the Mars atmosphere, as seen from outside (Fig. 8).

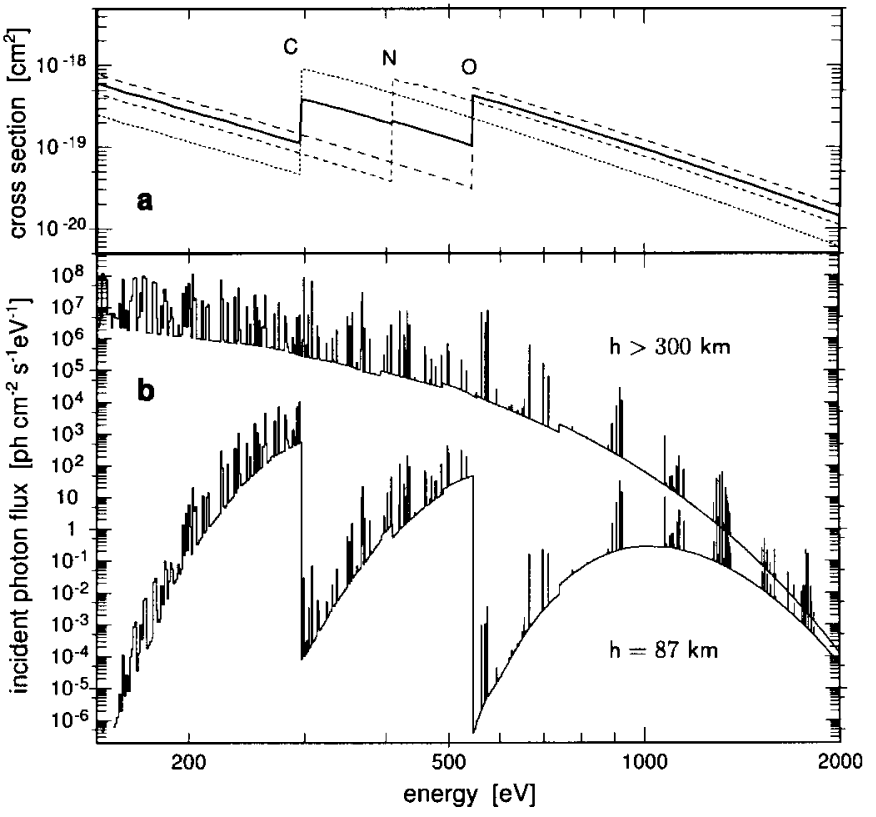

Fig. 9. a) Photoabsorption cross sections $\sigma_{\mathrm{C}}, \sigma_{\mathrm{N}}, \sigma_{\mathrm{O}}$ for $\mathrm{C}, \mathrm{N}$, and $\mathrm{O}$ (dashed lines), and $\sigma_{\mathrm{CNO}}$ for the chemical composition of the Mars atmosphere (solid line). b) Incident solar X-ray photon flux on top of the Mars atmosphere $(h>300 \mathrm{~km})$ and at $87 \mathrm{~km}$ height. The spectrum is plotted in $1 \mathrm{eV}$ bins. At $87 \mathrm{~km}$, it is considerably attenuated just above the $\mathrm{K}_{\alpha}$ absorption edges, recovering towards higher energies.

\subsection{Solar radiation}

The solar spectra for 2001 July 4 were derived from SOLAR 2000 (Tobiska et al. 2000) ${ }^{3}$. To improve the coverage towards energies $E>100 \mathrm{eV}$, synthetic spectra were computed with the model of Mewe et al. (1985) and aligned with the SOLAR 2000 spectra in the range $50-500 \mathrm{eV}$, by adjusting the temperature and intensity. This comparison yielded a fairly low average coronal temperature of only $\sim 80 \mathrm{eV}$. The adopted solar spectrum, scaled to the heliocentric distance of Mars, is shown in Fig. 9b (upper curve), with a bin size of $1 \mathrm{eV}$, which was used in order to preserve the spectral details.

\subsection{Model grid}

The high dynamic range in the optical depth of the Mars atmosphere requires a model with high spatial resolution. Figure 8 shows that the atmosphere becomes optically thick for X-rays with $E<1 \mathrm{keV}$ already at heights above $100 \mathrm{~km}$ during solar maximum (and above $90 \mathrm{~km}$ during solar minimum). This implies that most of the scattering takes place at heights where the latitudinal dependence of the atmospheric density is negligible. Thus, the volume elements need to be calculated only on a two dimensional grid (as in the case of Venus). For the calculation a grid of cubic volume elements with a side length of $1 \mathrm{~km}$ was used. The model atmosphere was traced from the surface (at $r=3393.4 \mathrm{~km}$ ) to a height of $300 \mathrm{~km}$. The simulated images were synthesized with $20 \mathrm{~km}$ resolution perpendicular to the line of sight. Details about the simulation itself can be found in Dennerl et al. (2002a).

\footnotetext{
3 Available at http://SpaceWx.com/
} 


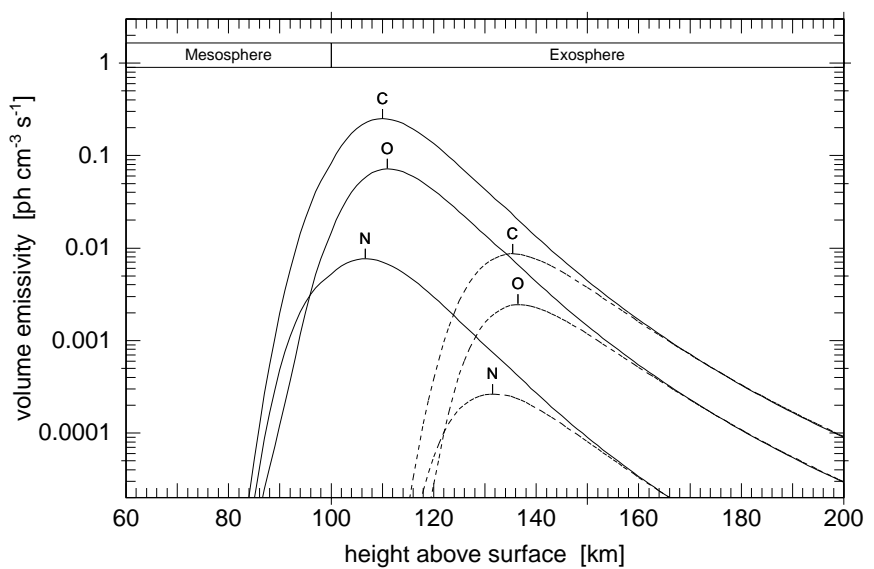

Fig. 10. Volume emissivities of $\mathrm{C}, \mathrm{N}$, and $\mathrm{O} \mathrm{K}_{\alpha}$ fluorescent photons at zenith angles of zero (subsolar, solid lines) and $90^{\circ}$ (terminator, dashed lines) for the incident solar spectrum of Fig. 9b. The height of maximum emissivity rises with increasing solar zenith angles because of increased path length and absorption along oblique solar incidence angles. In all cases maximum emissivity occurs in the exosphere, where the optical depth depends also on the solar cycle (Fig. 8).

\subsection{Planning the Mars observation}

The simulation program was already used for optimizing the time of the Mars observation. Although the closest approach of Mars to Earth, with a minimum distance of $0.45 \mathrm{AU}$, occurred on 22 June 2002, the Chandra observation was postponed by a few weeks. This decision was motivated by the fact that the simulation indicated a practically uniform X-ray brightness across the whole planet for this time (cf. Fig. 12), while for phase angles of $\sim 15^{\circ}$ and more, a diagnostically more valuable view was predicted, with a characteristic brightening on the sunward limb (Figs. 11a-c). The decision to postpone the Chandra observation was supported by the favorable fact that Mars was still approaching the perihelion of its orbit, so that its distance from Earth would increase only slightly to $0.46 \mathrm{AU}$. Furthermore, the small loss of $\mathrm{X}$-ray photons due to the reduced solid angle would be almost compensated by the fact that Mars would then be closer to the Sun and would intercept more solar radiation.

\section{Discussion}

\subsection{Morphology}

The simulation shows (Fig. 10) that the scattering of solar $\mathrm{X}$-rays takes place at heights above $\sim 80 \mathrm{~km}$ and is most efficient between $110 \mathrm{~km}$ (along the subsolar direction) and $136 \mathrm{~km}$ (along the terminator). This behaviour is similar to Venus, where the volume emissivity was found to peak between $122 \mathrm{~km}$ and $135 \mathrm{~km}$ (Dennerl et al. 2002a). The fact that the volume emissivity for $\mathrm{C}$ is considerably higher than that of $\mathrm{O}$ is a direct consequence of the unusually soft solar spectrum during the Mars observation (cf. Fig. 9b). During the Venus observation, the photon fluxes from $\mathrm{C}$ and $\mathrm{O}$ were comparable.

Figures $11 \mathrm{a}-\mathrm{c}$ show the simulated images of Mars at the $\mathrm{K}_{\alpha}$ fluorescence lines of $\mathrm{C}, \mathrm{N}$, and $\mathrm{O}$, for a phase angle of

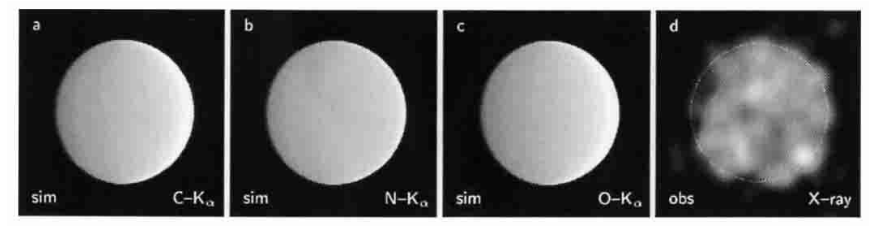

Fig. 11. a)-c) Simulated $\mathrm{X}$-ray images of Mars at $\mathrm{C}-\mathrm{K}_{\alpha}, \mathrm{N}-\mathrm{K}_{\alpha}$, and $\mathrm{O}-\mathrm{K}_{\alpha}$, for a phase angle of $18^{\circ} .2$. The $\mathrm{X}$-ray flux is coded in a linear scale, extending from zero (black) to a) $5.0 \times 10^{5} \mathrm{ph} \mathrm{cm}^{-2} \mathrm{~s}^{-1}$, b) $1.1 \times 10^{4} \mathrm{ph} \mathrm{cm}^{-2} \mathrm{~s}^{-1}$, and c) $1.5 \times 10^{5} \mathrm{ph} \mathrm{cm}^{-2} \mathrm{~s}^{-1}$, (white). All images show some limb brightening, especially at $\mathrm{C}-\mathrm{K}_{\alpha}$ and $\mathrm{O}-\mathrm{K}_{\alpha}$. d) Observed X-ray image, accumulated in the energy range 0.4-0.7 and smoothed with a Gaussian filter with $\sigma=1$ '.2. The circle indicates the geometric size of Mars. This image is dominated by $\mathrm{O}-\mathrm{K}_{\alpha}$ fluorescence photons. Although the brightness fluctuations are mainly caused by photon statistics and are not significant, there is evidence for limb brightening on the right-hand side (cf. Fig. 3b).

18.2. Although Mars was almost fully illuminated, there is already some brightening on the more sunward limb evident, especially at $\mathrm{C}$ and $\mathrm{O}$, accompanied by a fading on the opposite limb. While a direct comparison with the observed Mars image (Fig. 11d) suffers from low photon statistics, a similar trend can be seen in the surface brightness profiles (Fig. 3b). Thus, the expected limb brightening (Sect. 4.5) was actually observed. The reason for the limb brightening and the different appearance of Mars in the three fluorescent lines is very similar to the case of Venus, and a discussion can be found in Dennerl et al. (2002a). The close match between the simulated and observed morphology is an argument in favor of X-ray fluorescence as the dominant process responsible for the $\mathrm{X}$-ray radiation of Mars. With simulations based on charge exchange interactions (Sect. 5.5), Holmström et al. (2001) obtained a competely different X-ray morphology.

\subsection{Spectrum, $X$-ray flux and luminosity}

The ACIS-I spectrum of Mars is dominated by a single narrow emission line. Although this line appears at $0.65 \mathrm{keV}$, it is most likely the $\mathrm{O}-\mathrm{K}_{\alpha}$ fluorescence line at $0.53 \mathrm{keV}$. This conclusion is motivated by the fact that in the case of Venus a similar line was observed at $0.6 \mathrm{keV}$ with the same detector (Fig. 9 in Dennerl et al. 2002b), which could be uniquely identified to be at $0.53 \mathrm{keV}$ by the additional LETG observation. The apparent energy shift is most likely caused by optical loading, a superposition of the charges released by $0.53 \mathrm{keV}$ photons and optical photons, during the $3.2 \mathrm{~s}$ exposure of each CCD frame.

The simulated images can be used to estimate the expected photon flux from the whole visible side of Mars. For the three energies the following values are obtained: $f_{\mathrm{C}}=2.3 \times 10^{-4}$, $f_{\mathrm{N}}=5.0 \times 10^{-6}, f_{\mathrm{O}}=7.1 \times 10^{-5} \mathrm{ph} \mathrm{cm}^{-2} \mathrm{~s}^{-1}$. While the $\mathrm{C}$ and $\mathrm{N}$ emission lines are outside the energy range of ACIS$\mathrm{I}$, a direct comparison is possible for $\mathrm{O}-\mathrm{K}_{\alpha}$, where a flux of $(6.3 \pm 0.8) \times 10^{-5} \mathrm{ph} \mathrm{cm}^{-2} \mathrm{~s}^{-1}$ was observed (Sect. 3.2). This flux is reduced to $(5.4 \pm 0.9) \times 10^{-5} \mathrm{ph} \mathrm{cm}^{-2} \mathrm{~s}^{-1}$, if an additional bremsstrahlung component is added. In view of all the general uncertainties, these values are in good agreement with each other. 

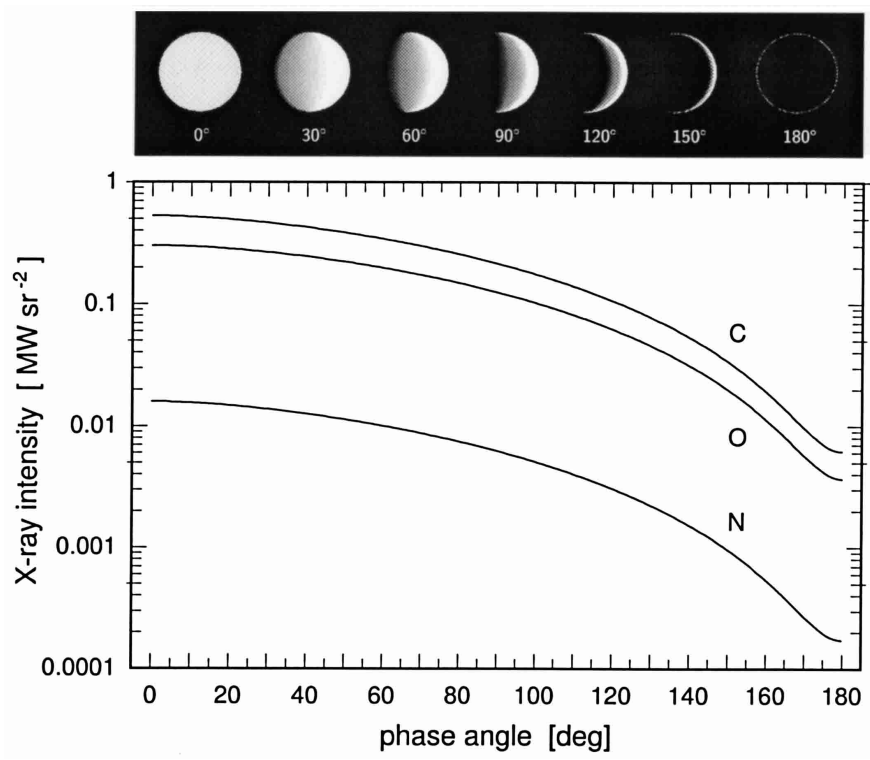

Fig. 12. X-ray intensity of Mars as a function of phase angle, in the fluorescence lines of C, N, and O, for the conditions on 4 July 2001. The images at top, all displayed in the same intensity coding, illustrate the appearence of Mars at $\mathrm{O}-\mathrm{K}_{\alpha}$ for selected phase angles.

Conversion of the observed flux to the luminosity requires knowledge about the angular distribution of the scattered photons. For this purpose, $\mathrm{X}$-ray intensities were determined from simulated Mars images, computed for phase angles from $0^{\circ}$ to $180^{\circ}$ in steps of $1^{\circ}$ (Fig. 12). By spherically integrating these intensities for the three energies over phase angle, the following luminosities are obtained from the simulation: $2.9 \mathrm{MW}$ for $\mathrm{C}$, 0.1 MW for N, and 1.7 MW for O. The total X-ray luminosity of Mars, 4.7 MW (or $\sim 3.6 \pm 0.6 \mathrm{MW}$ when adjusted to the observed $\mathrm{O}-\mathrm{K}_{\alpha}$ flux), agrees well with the prediction of Cravens \& Maurellis (2001), who estimated a luminosity of 2.5 MW due to X-ray fluorescence, with an uncertainty factor of about two. This is another argument in favor of X-ray fluorescence.

Compared to its optical flux, the X-ray flux of Mars is very low: the visual magnitude -2.1 mag corresponds to an optical flux $f_{\mathrm{opt}}=1.8 \times 10^{-4} \mathrm{erg} \mathrm{cm}^{-2} \mathrm{~s}^{-1}$. Adopting a total $\mathrm{X}$-ray flux $f_{\mathrm{x}}=9 \times 10^{-14} \mathrm{erg} \mathrm{cm}^{-2} \mathrm{~s}^{-1}$, a ratio $f_{\mathrm{x}} / f_{\text {opt }}=5 \times 10^{-10}$ follows. This is similar to the value $2 \times 10^{-10}$ observed for Venus (Dennerl et al. 2002a). In the case of X-ray fluorescence, the $f_{\mathrm{x}} / f_{\text {opt }}$ ratio of Mars is generally expected to exceed that of Venus, because the optical albedo of Mars is lower than that of Venus, while their X-ray albedos are comparable. Both ratios, however, are expected to vary with time, in response to the temporarily variable solar X-ray flux (cf. Fig. 13).

\subsection{The ROSAT observation of Mars in 1993}

Mars was observed with the ROSAT Position Sensitive Proportional Counter (PSPC) from 10-13 April 1993 on three occasions, for 1294 s, 2124 s, and 1099 s, respectively. As the pointing direction of the satellite was kept fixed during these observations, Mars was located at different positions in the $2^{\circ}$ PSPC field of view (FOV). During the first and third exposure, Mars was partially obscured by a radial strut of the PSPC

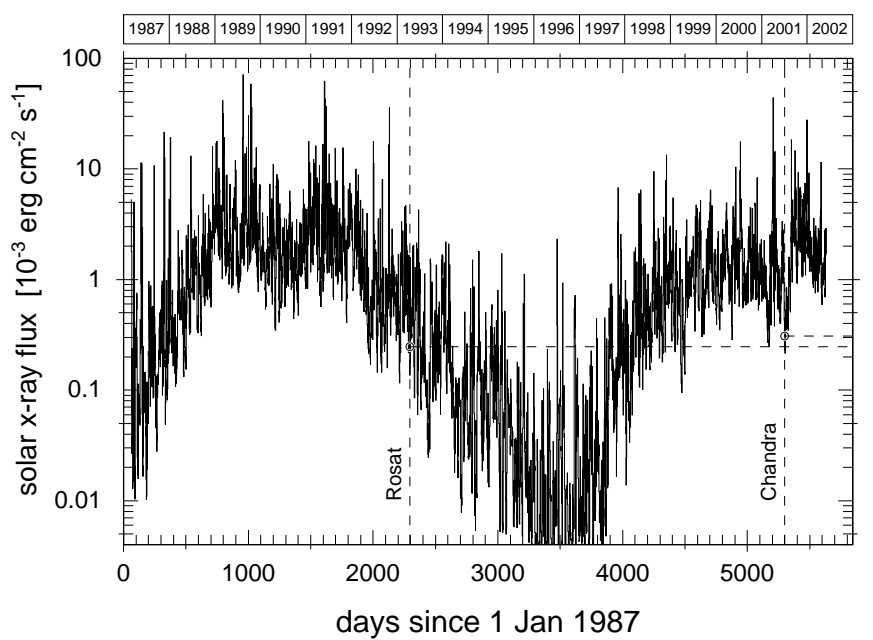

Fig. 13. 1-8 ̊̊ (1.55-12.4 keV) solar X-ray flux at $1.0 \mathrm{AU}$, measured with GOES-7 (before March 1995) and GOES-8 (afterwards). During the ROSAT and Chandra observations (indicated by dashed vertical lines), the solar X-ray flux was similar.

support structure, and was furthermore placed so much in the outer parts of the FOV, where the point spread function was severely degraded, that only the second observation is suited for a sensitive search for any X-ray emission. During this observation, Mars was at a heliocentric distance of $1.67 \mathrm{AU}$. The constraint to observe it at an elongation close to $90^{\circ}$ implied a fairly large geocentric distance from Earth, 1.32 AU, so that Mars appeared as a disk with a diameter of only $7{ }^{\prime \prime} 1$, seen at a phase angle of $37^{\circ} .0$. Mars was not detected in this observation. From the second PSPC exposure, a $3 \sigma$ upper limit of $4 \times 10^{-3}$ counts $\mathrm{s}^{-1}$ can be derived in the energy range $0.1-$ $0.9 \mathrm{keV}$, for a circle around the nominal position of Mars with a radius of $1^{\prime}$.

How does this non-detection with ROSAT compare with the information which is now available on the X-ray properties of Mars? According to the SOLAR 2000 data for 13 April 1993, the solar X-ray flux at 1 AU was about $30 \%$ fainter than during the Chandra observation (cf. Fig. 13), but showed a similar spectral shape. Taking also the larger heliocentric distance into account, Mars received about half of the solar flux. The simulation then yields the following number of expected counts for the second PSPC observation: 5 counts at $\mathrm{C}, 0.003$ counts at $\mathrm{N}$, and 0.3 counts at $\mathrm{O}$. The total number of expected counts, $\sim 5$, is somewhat lower than the local background in the detect cell ( 8). This suggests that the X-ray signal of Mars was just below the sensitivity limit of the ROSAT observation.

\subsection{Temporal variability and the dust storm of 2001}

Scattering of solar X-rays on very small dust particles was one of the early suggestions for explaining the X-ray emission from comets. Wickramasinghe \& Hoyle (1996) noted that Xrays can be efficiently scattered by dust particles, if their size is comparable to the X-ray wavelength. Such attogram dust particles $\left(\sim 10^{-18} \mathrm{~g}\right)$ would be difficult to detect by other means. It might be possible that such particles are present in the upper Mars atmosphere, in particular during episodes of global dust storms. 


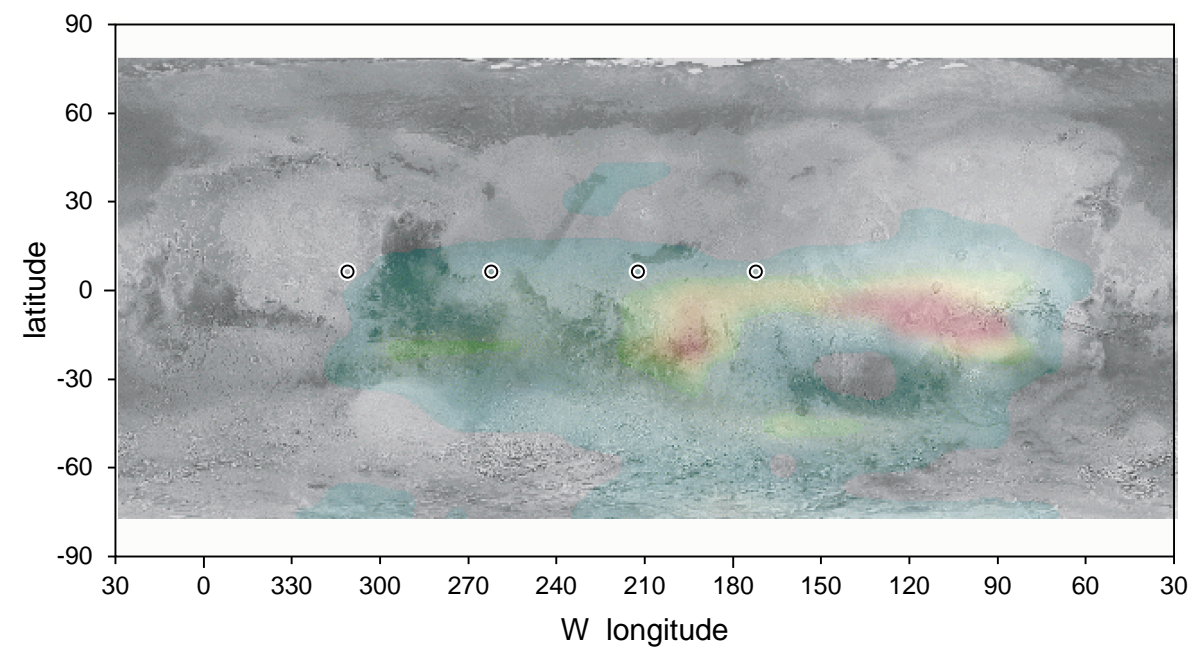

Fig. 14. Mars during the Chandra observation. Grey: viking map of Mars, courtesy NASA/JPL/Caltech (http://maps.jpl.nasa.gov/). For a better comparison with the simulated and observed images, this map was shifted by $150^{\circ}$ in longitude. The circles indicate the central positions of the images in Figs. 15 and 16. Color: dust optical depth, derived with the Thermal Emission Spectrometer on Mars Global Surveyor from 12 orbits on 4 July 2001 (adopted from Smith et al. 2002). Green, yellow, and red colours mark dust optical depths of 1.0, 1.5, and $\geq 2.0$. In all coloured areas the atmosphere was very dusty and in a typical dust storm condition.
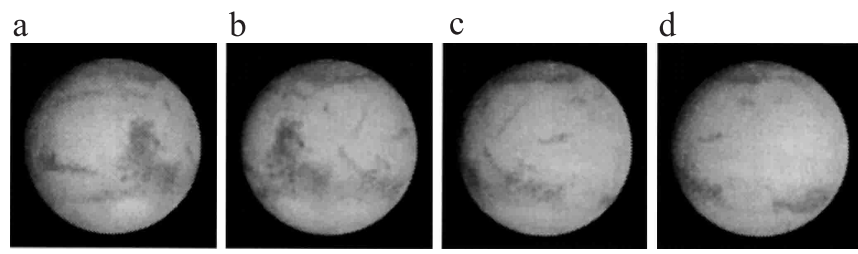

Fig. 15. Simulated "clear" views of Mars, obtained from http://space.jpl.nasa.gov/. In order to facilitate comparison with the map (Fig. 14), they were arranged with decreasing central meridian. a) 04 July 2001, 21:40 UT, LCM $=311^{\circ}$ b) 01 July $2001,16: 30 \mathrm{UT}, \mathrm{LCM}=262^{\circ}$ c) 04 July 2001, 14:55 UT, $\mathrm{LCM}=212^{\circ}$ d) 04 July 2001, 12:10 UT, LCM $=172^{\circ}$.

Incidentally, on June 26 a local dust storm on Mars originated and expanded quickly, developing into a planetencircling dust storm by July 11 (Smith et al. 2002). Such dust storms have been observed on roughly one-third of the perihelion passages during the last decades, but never so early in the Martian year. On July 4, this very vigorous storm had covered roughly one hemisphere (Fig. 14). This hemisphere happened to be visible at the beginning of the Chandra observation. By the end of the observation, which covered one third of a Mars rotation, this hemisphere had mainly rotated away from our view (Figs. 14-16). Thus, a comparison of the Chandra data from both regions should reveal any influence of the dust storm on the X-ray flux.

There is, however, no change in the mean X-ray flux between the first and second half of the observation, where 150 and 157 photons were detected, respectively. This implies that, if attodust particles are present in the upper Mars atmosphere, the dust storm did not lead to a local increase in their density, high enough to modify the observed $\mathrm{X}$-ray flux significantly. No statement, however, can be made about the situation below $\sim 80 \mathrm{~km}$, as the solar X-rays do not reach these atmospheric layers (Fig. 10). While the general presence of some attodust in the upper atmosphere cannot be ruled out by the Chandra observation, the fact that the ACIS-I spectrum of Mars is dominated by a single emission line (Fig.4) shows that any
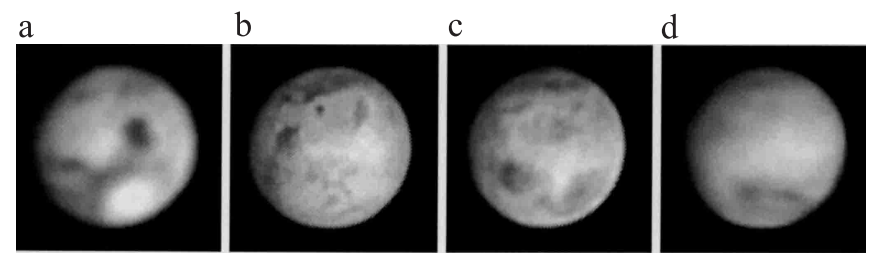

Fig. 16. Optical images of Mars, taken during the Chandra observation (except $b$, which was taken three days earlier), and arranged as in Fig. 15. a) 04 July 2001, 21:38 UT, LCM $=311^{\circ}$ courtesy B. FlachWilken, Germany b) 01 July 2001, 16:30 UT, LCM $=262^{\circ}$, courtesy T. Wei Leong, Singapore c) 04 July 2001, 14:55 UT, LCM $=212^{\circ}$, courtesy Y. Morita, Japan, CMO Archives of the OAA Mars Section d) 04 July 2001, 12:10 UT, LCM $=172^{\circ}$, courtesy G. Garradd, Australia. Note how significantly the surface markings were changed by the dust storm, particularly at longitudes $\ell_{\mathrm{w}}<270^{\circ}$ b) -d), while the hemisphere at $\ell_{\mathrm{w}}>270^{\circ}$ a) was much less affected.

contribution of such particles to the X-ray flux from Mars must be small compared to fluorescence, even in the process of a developing global dust storm.

\subsection{The X-ray halo}

Although the significance of a soft X-ray halo around Mars is only $\sim 4 \sigma$, its spectrum is clearly different from that of Mars itself (Fig. 4), ruling out the possibility that the halo is an instrumental artefact, related to the point spread function of the $\mathrm{X}$-ray telescope. It can also be ruled out that the halo is caused by the vignetting of the telescope, because the $11^{\prime \prime}<r<30^{\prime \prime}$ halo contains $2.1 \pm 0.3$ times more photons than the same area in the $50^{\prime \prime}<r<100^{\prime \prime}$ background region, while vignetting would affect the number of photons by less than $5 \%$ at energies below $1.5 \mathrm{keV}$. Furthermore, the halo cannot be an artefact of exposure variations introduced by removing the point sources, because no gradient in the surface brightness is observed at $E=1.5-10.0 \mathrm{keV}$ (Fig. 3b). Therefore, the following discussion assumes that the $\mathrm{X}$-ray halo is real.

While there is a lot of evidence that the X-rays from Mars are predominantly caused by fluorescent scattering of solar 
$\mathrm{X}$-rays in its upper atmosphere, there is the possibility of an additional source of $\mathrm{X}$-ray emission. When highly ionized heavy ions in the solar wind encounter atoms in the exosphere of Mars, they become discharged and may emit X-rays. This is the process which was found to be responsible for the $\mathrm{X}$ ray emission of comets (Cravens 1997; Lisse et al. 2001). Its consequences for the $\mathrm{X}$-ray emission of Mars were already investigated by several authors. Cravens (2000b) predicted an Xray luminosity of $\sim 0.01 \mathrm{MW}$. Krasnopolsky (2000) estimated an X-ray emission of $\sim 4 \times 10^{22} \mathrm{ph} \mathrm{s}^{-1}$. Adopting an average photon energy of $200 \mathrm{eV}$ (e.g. Cravens 1997), this corresponds to an X-ray luminosity of 1.3 MW. Holmström et al. (2001) computed a total X-ray luminosity of Mars due to charge exchange (within 10 Mars radii) of $1.5 \mathrm{MW}$ at solar maximum, and 2.4 MW at solar minimum.

For the X-ray halo observed within 3 Mars radii, excluding Mars itself, the Chandra observation yields a flux of $(0.9 \pm 0.4) \times 10^{-14} \mathrm{erg} \mathrm{cm}^{-2} \mathrm{~s}^{-1}$ in the energy range $E=$ $0.5-1.2 \mathrm{keV}$ (Sect. 3.2). Assuming isotropic emission, this flux corresponds to a luminosity of $0.5 \pm 0.2 \mathrm{MW}$. This value agrees well with the predictions of Krasnopolsky (2000) and Holmström et al. (2001), in particular when the spectral shape is extrapolated to lower energies ${ }^{4}$.

In addition to the luminosity, there is another argument in favor of the idea that the X-ray halo may be the signature of charge exchange. Although this process produces a spectrum consisting of many narrow emission lines, the overall properties can be approximated by $0.2 \mathrm{keV}$ thermal bremsstrahlung emission (Wegmann et al. 1998), and the spectrum of the $\mathrm{X}$-ray halo agrees very well with such a model. Also the spectrum of Mars itself shows evidence for an emission component with this spectral shape (Fig. 4). The Chandra data, however, indicate that the surface brightness of this component in the spectrum of Mars is by one order of magnitude higher than that in the halo, averaged from one to three Mars radii. This is different from the result of computer simulations by Holmström et al. (2001), where the surface brightness in front of Mars is lower than in the halo. In these simulations an empirical model of the proton flow near Mars was used, where the proton flux decreases strongly at the "magnetopause", at $\sim 680 \mathrm{~km}$ height. The fact that the surface brightness at the center was observed to be higher than expected could be an indication that the dilution of the heavy ion flux near the "magnetopause" might be less pronounced than assumed in the model. It has to be stressed, however, that the observational evidence for any emission component in addition to the $\mathrm{X}$-ray fluorescence is near the sensitivity limit of the observation and that any statement about observational properties may be subject to considerable uncertainties.

\section{Summary and conclusions}

The Chandra observation clearly shows that Mars is an Xray source. The luminosity, the X-ray spectrum, the morphology and the time variability are all consistent with fluorescent

\footnotetext{
${ }^{4}$ It has, however, be kept in mind that there may be an additional uncertainty in these values, because the $\mathrm{O}-\mathrm{K}_{\alpha}$ fluorescence line was found to be displaced by $\sim 120 \mathrm{eV}$ (Sect. 5.2).
}

scattering of solar X-rays on oxygen atoms in the Mars atmosphere at heights above $\sim 80 \mathrm{~km}$ as the main process for the observed radiation. No evidence for dust-related X-ray emission was found, despite the onset of a global dust storm, which had covered roughly one hemisphere at the time of the observation. Differential measurements between the hemisphere affected by the dust storm and the quiet hemisphere showed no significant difference in the X-ray flux. There is, however, some evidence for an additional source of $\mathrm{X}$-ray emission, indicated by a faint X-ray halo which can be traced to about three Mars radii, and by an additional component in the $\mathrm{X}$-ray spectrum of Mars, which has a similar spectral shape as the halo. Within the available limited statistics, the spectrum of this component can be characterized by $0.2 \mathrm{keV}$ thermal bremsstrahlung emission. The spectral shape and the luminosity are indicative of charge exchange interactions between highly charged heavy ions in the solar wind and exospheric hydrogen and oxygen around Mars. The significance of the halo, however, is only $4 \sigma$, and additional observations will be needed for further studies. Such observations would also provide additional information about the temporal properties of the exosphere of Mars, in particular with respect to the solar cycle.

Acknowledgements. It is a great pleasure to thank S. Wolk for his support in planning this observation, B. Aschenbach, V. Burwitz, J.Englhauser and C. Lisse for stimulating discussions, and G. Garradd, Y. Morita, T. Wei Leong and B. Flach-Wilken for providing the optical images. SOLAR 2000 Research Grade v1.15 historical irradiances are provided courtesy of W. Kent Tobiska and SpaceWx.com. These historical irradiances have been developed with funding from the NASA UARS, TIMED, and SOHO missions. The SOHO CELIAS/SEM data were provided by the USC Space Sciences Center. SOHO is a joint European Space Agency, United States National Aeronautics and Space Administration mission. The Yohkoh image was obtained from the Yohkoh Data Archive Centre (YDAC). Yohkoh is a mission of the Japanese Institute for Space and Astronautical Science.

\section{References}

Cravens, T. E. 1997, Geophys. Res. Lett., 24, 105

Cravens, T. E. 2000a, ApJ, 532, L153

Cravens, T. E. 2000b, Adv. Space Res., 26, 1443

Cravens, T. E., \& Maurellis, A. N. 2001, Geophys. Res. Lett., 28, 3043

Dennerl, K., Burwitz, V., Englhauser, J., Lisse, C., \& Wolk, S. 2002a, A\&A, 386, 319

Dennerl, K., Burwitz, V., Englhauser, J., Lisse, C., \& Wolk, S. 2002b, in New Visions of the Universe in the XMM-Newton and Chandra Era, ed. F. Jansen, ESA SP-488 [astro-ph/0204263]

Dennerl, K., Englhauser, J., \& Trümper, J. 1997, Science, 277, 1625

Elsner, R. F., Gladstone, G. R., Waite, J. H., et al. 2002, ApJ, 572, 1077

Friedman, H., Lichtman, S. W., \& Byram, E. T. 1951, Phys. Rev., 83, 1025

Gorenstein, P., Golub, L., \& Bjorgholm, P. 1974, Moon, 9, 129

Grader, R. J., Hill, R. W., \& Seward, F. D. 1968, J. Geophys. Res., 73, 7149

Holmström, M., Barabash, S., \& Kallio, E. 2001, Geophys. Res. Lett., 28, 1287

Krasnopolsky, V. 2000, Icarus, 148, 597 
Krause, M. O. 1979, J. Phys. Chem. Ref. Data, 8, 307

Lisse, C. M., Christian, D. J., Dennerl, K., et al. 2001, Science, 292, 1343

Lisse, C. M., Dennerl, K., Englhauser, J., et al. 1996, Science, 274, 205

Metzger, A. E., Gilman, D. A., Luthey, J. L., et al. 1983, J. Geophys. Res., 88, 7731

Mewe, R., Gronenschild, E. H. B. M., \& van den Oord, G. H. J. 1985, A\&AS, 62, 197

Mumma, M. J., Krasnopolsky, V. A., \& Abbott, M. J. 1997, ApJ, 491, L125

Ness, J.-U., \& Schmitt, J. H. M. M. 2000, A\&A, 355, 394
Reilman, R. F., \& Manson, S. T. 1979, ApJS, 74, 815

Schmitt, J. H. M. M., Snowden, S. L., Aschenbach, B., et al. 1991, Nature, 349, 583

Sehnal, L. 1990a, Bull. Astron. Inst. Czechosl., 41, 115

Sehnal, L. 1990b, Bull. Astron. Inst. Czechosl., 41, 108

Smith, M. D., Conrath, B. J., Pearl, J. C., \& Christensen, P. R. 2002, Icarus, 157, 259

Tobiska, W. K., Woods, T., Eparvier, F., et al. 2000, J. Atm. Sol. Terr. Phys., 62, 1233

Wegmann, R., Schmidt, H. U., Lisse, C. M., Dennerl, K., \& Englhauser, J. 1998, Planet. Space Sci., 46.5, 603

Wickramasinghe, N. C., \& Hoyle, F. 1996, Ap\&SS, 239, 121 